\title{
In Search of Concrete Outcomes-A Systematic Review on the Effectiveness of Educational Interventions on Reducing Acute Occupational Injuries
}

\author{
Jim Li 1,2,*(D), Max Pang ${ }^{1}$, Jennifer Smith ${ }^{2}$, Colleen Pawliuk ${ }^{3} \mathbb{D}$ and Ian Pike 2,3,4 (D) \\ 1 MD Undergraduate Program, Faculty of Medicine, University of British Columbia, \\ Vancouver, BC V6T 1Z3, Canada; mpang@alumni.ubc.ca \\ 2 BC Injury Research and Prevention Unit, Faculty of Medicine, University of British Columbia, \\ BC Children's Hospital, Vancouver, BC V6H 3V4, Canada; jsmith@bcchr.ca (J.S.); ipike@bcchr.ca (I.P.) \\ 3 BC Children's Hospital Research Institute, Faculty of Medicine, University of British Columbia, \\ BC Children's Hospital, Vancouver, BC V6H 3V4, Canada; cpawliuk@bcchr.ca \\ 4 Department of Pediatrics, Faculty of Medicine, University of British Columbia, \\ Vancouver, BC V6H 3V4, Canada \\ * Correspondence: jimzhanghao.li@alumni.ubc.ca; Tel.: +1-778-893-2655
}

Received: 11 August 2020; Accepted: 18 September 2020; Published: 20 September 2020

\begin{abstract}
Education is a common strategy used to prevent occupational injuries. However, its effectiveness is often measured using surrogate measures instead of true injury outcomes. To evaluate the effectiveness of workplace educational interventions, we selectively analyzed studies that reported injury outcomes (PROSPERO ID: CRD42019140631). We searched databases for peer-reviewed journal articles and sources of grey literature such as abstracts, registered trials, and theses published between 2000 and 2019. Studies on educational interventions that reported fatal or non-fatal occupational injury outcomes were selected. Two reviewers independently and in duplicate screened the studies, extracted data, and assessed risk of bias. Heterogeneity in the data precluded meta-analysis, and the results were reviewed narratively. In total, 35 studies were included. Of which, 17 found a significant reduction in injuries, most of which featured a multifaceted approach or non-didactic education. The remaining studies either described equivocal results or did not report statistical significance. Overall, interventions in the manufacturing industry were more effective than those in the construction sector. Risk of bias among included studies was moderate to high. In conclusion, educational interventions could be an effective part of multifaceted injury prevention programs. However, over-reliance on didactic education alone is not advised.
\end{abstract}

Keywords: accident prevention; educational intervention; injury prevention; occupational injuries; preventive medicine; public health; safety management; safety training; systematic review

\section{Introduction}

Occupational injuries are a major threat to the health and well-being of workers. Each year, an estimated 380,000 workers lose their lives due to workplace injuries globally, and over 370,000,000 suffer non-fatal injuries [1]. In 2015, the International Labour Organization estimated that a total of USD \$2.8 trillion is lost each year due to the direct and indirect effects of occupational hazards globally, accounting for $4 \%$ of the world's GDP $[2,3]$.

Many prevention strategies have been implemented in recent decades in an effort to stem occupational injury levels. These initiatives can be broadly categorized into the "Three E's of Injury 
Prevention", education, enforcement, and engineering [4]. Education refers to providing information and training to elicit behavioral change, enforcement includes laws and regulations to deter unsafe practices, and engineering entails modifying the environment and/or the task to enhance safety. Since educational interventions theoretically work by changing employee attitudes, behavior, and knowledge, their effectiveness is often assessed based on such surrogate measures, instead of direct injury outcomes [5]. This assumption has been criticized for being overly simplistic, as surrogate measures are not necessarily representative of true injury rates, thereby making the data difficult to interpret with certainty [6,7]. Various reviews on educational interventions have been challenged to make concrete recommendations due to the lack of studies that report injury outcomes [6-9]. This highlights a gap in the literature, as the true effectiveness of education on reducing workplace injuries remains unclear.

Although some previous systematic reviews have been selective, by only including studies with injury outcomes, they are limited to specific industries, such as construction or agriculture $[10,11]$, which precludes generalization of the results to occupational injuries as a whole. Therefore, we endeavored to systematically review studies across all industries describing workplace educational interventions aimed at preventing occupational injuries, which also reported worker safety outcomes.

\section{Materials and Methods}

The review protocol is registered in PROSPERO (ID: CRD42019140631). The review was conducted in accordance with the preferred reporting items for systematic reviews and meta-analyses (PRISMA) guideline [12].

\subsection{Types of Studies}

Randomized controlled trials (RCTs), non-randomized controlled studies (NRSs), and uncontrolled before-and-after trials (uCBAs) were eligible for inclusion in this review. We expected a limited number of RCTs due to various logistical and ethical barriers inherent within the context of workplace health and safety education initiatives. For instance, educational interventions using individual-level randomization in the workplace may be prone to contamination between groups, since workers can freely communicate amongst each other. Additionally, workplace-based cluster randomization faces challenges associated with recruiting a sufficiently large sample to be robust against confounding variables. Therefore, we decided to include a number of non-randomized study designs, such as controlled before-and-after studies (CBA), interrupted time studies (ITS), historically controlled studies, prospective cohort studies, and uncontrolled before-and-after studies (uCBAs).

We defined a CBA study as a controlled trial in which the experimental and control groups were not assigned in a random fashion. A historically controlled study is similar to a CBA study, with the difference that the control was a group of participants in the past. In the absence of a control group, if a study measured three time points before and after the intervention to analyze for temporal trends, then it qualified as an ITS study. A prospective cohort study was defined as a study in which the investigators enrolled participants after exposure to a factor associated with the outcome, but before any outcome(s) of interest had developed. Finally, an uCBA study implements an intervention without including a control group [13].

Our decision to include uncontrolled before-and-after studies was due to three reasons. First, in the occupational context, controlled trials may be difficult to perform. Second, since the literature connecting educational interventions to injury outcomes is already limited to begin with, over-restriction of our search criteria may have limited our ability to draw any conclusions at all. Third, we anticipated that a sizeable proportion of the literature on this topic may be the result of quality improvement projects which were later published. By including these uncontrolled trials, we could gain additional insight into educational interventions used in a practical context. 


\subsection{Types of Participants}

This review included studies of paid adult ( $\geq 18$ years of age) employees from all industries. Temporary workers or workers employed outside of a legal contract were also included.

\subsection{Types of Interventions}

Educational interventions implemented with the intention of preventing occupational injuries were included. Multifaceted interventions with a prominent educational component were included as well. We included didactic education in addition to non-traditional methods, such as interactive discussions, peer feedback, hazard recognition, and social marketing/awareness campaigns.

\subsection{Types of Outcome Measures}

We included studies that measured the change in fatal or nonfatal injury rate following educational intervention(s). As an operational definition, we used the following modified definition from The Injury Chartbook by the World Health Organization (WHO) [14,15], "occupational injury is a body lesion at the organic level, resulting from acute exposure to energy in the work environment (mechanical, thermal, electrical, chemical or radiant) in amounts that exceed the threshold of physiological tolerance. In some cases (e.g., drowning, strangulation, freezing), the injury results from an insufficiency of a vital element." Since we anticipated that operational definitions for injury would vary by study, we considered all reasonably congruent definitions. All collection methods of quantifiable injury data were eligible for inclusion. These included, but were not limited to, self-report, company databases, and workers' compensation records.

\subsection{Exclusion Criteria}

We excluded studies published before 2000 to ensure the recency of our results. The year 2000 was selected due to the rapid automation of industries which caused substantial changes in the proportion of white-collar, operative/laborer/service, and agricultural workers that did not decelerate until $2000[16,17]$. This coincided with a shifting paradigm for occupational safety education, which better reflected the needs of the changing workplace [18,19]. We also excluded non-English studies due to limited translation resources. Studies that measured injury rates outside of the occupational context were excluded. For our study, "occupational context" is defined as both the workplace and commute to and from work. Further, we excluded occupational diseases, infections, intentional injuries, and musculoskeletal disorders due to chronic exposure.

\subsection{Information Sources}

We searched the following electronic databases up to May 2019: Embase, Ovid MEDLINE, NIOSHTIC, Web of Science Core Collection, and the Cochrane Central Register of Controlled Trials (CENTRAL). In order to avoid publication bias favoring positive results, grey literature such as theses, abstracts, and registered clinical trials were also included in our study [20]. We searched Google Scholar, Google, WorldCat, ProQuest, NDLTD (Networked Digital Library of Theses and Dissertations), and ClinicalTrials.gov for grey literature. We also manually searched the reference lists of all included studies to locate additional papers. All searches were conducted with the guidance of a biomedical librarian. The general PICO (population, intervention, context, and outcome) search structure for the databases is shown in Table 1; the detailed search strategy is listed in Appendix A. 
Table 1. General search topics included in the final PICO (population, intervention, context, and outcome) framework.

\begin{tabular}{cccc}
\hline Population/Problem & Intervention & Context & Outcome \\
\hline Injury & Education & Work & Injury prevention \\
Wounds & Program & Occupation & Injury reduction \\
Fatality & Social Marketing & Employee & Insurance claims \\
Disability & Primary Prevention & Industry & Absenteeism \\
\hline
\end{tabular}

\subsection{Selection of Studies}

The titles and abstracts of potentially relevant studies were screened by two review authors independently and in duplicate. Disagreements about inclusion occurred in less than $5 \%$ of all records screened, and were resolved by discussion until consensus was reached. In the case of a disagreement that could not be resolved by discussion, a third author made the final decision. Full text articles of the shortlisted studies were then reviewed independently and in duplicate by two authors against the inclusion and exclusion criteria. The same process for resolving disagreements was applied, and reasons for exclusion were documented. Reference lists of included papers and relevant systematic reviews were screened to identify additional studies. The titles of relevant grey literature were searched on Google Scholar to potentially locate their respective full papers.

\subsection{Data Extraction and Management}

Two review authors independently extracted data from all included articles. We designed a data extraction form in order to standardize the process between the two reviewers. The following data were collected: study design, setting (nationality and industry, as defined by the North American Industry Classification System [21]), participants (sample size, occupation, and distribution between experimental and control groups), description of the intervention(s), timeline of intervention, outcome (rate ratio, difference in means, etc.), sources of funding, and conflicts of interest.

\subsection{Assessment of Risk of Bias in Included Studies}

Two review authors assessed risk of bias in all included studies independently and in duplicate. The quality of RCTs was assessed using the revised Cochrane risk-of-bias tool for randomized trials (RoB 2) [22]. Quality of non-randomized controlled studies was assessed using the Cochrane risk of bias in non-randomized studies of interventions (ROBINS-I) [23]. Disagreements arose in about $10 \%$ of assessments but were all resolved by discussion without resorting to a third reviewer. The risk of bias for conference abstracts could not be assessed due to limited information, and thereby they were categorized as "uncertain risk of bias." Uncontrolled before-and-after trials were all categorized as "high risk of bias" since they are especially prone to confounding factors and regression to the mean [24].

\subsection{Data Synthesis}

Meta-analysis was not attempted due to heterogeneity in the target populations and study designs, as well as an insufficient number of studies from most industries. Since each industry has its unique profile of injury mechanisms and risk factors, we expected the nature of educational interventions to vary accordingly [25]. Therefore, qualitative assessment was performed at an industry-level, by considering the number and quality of studies, effect significance, and type of education involved. Included studies with a "critical" risk of bias were not factored into any narrative synthesis, as per Cochrane guidelines [23]. 


\section{Results}

\subsection{Results of the Search}

The search yielded 4492 records, of which 3973 were from electronic databases and 519 were identified through sources of grey literature. (Later manual checking of reference lists from relevant systematic reviews and included studies yielded an additional 5 records.) After removing the duplicates, 3613 remained. After screening their titles and abstracts, 48 studies were shortlisted for full-text review. Of these 48 papers, 38 met inclusion criteria (Figure 1), and 10 were excluded. The reasons for exclusions are listed in Appendix B. The 38 included papers represented 35 unique studies. In cases where multiple papers described the same study, only the most comprehensive publication was retained for further analysis. The redundant papers are also listed in Appendix B.

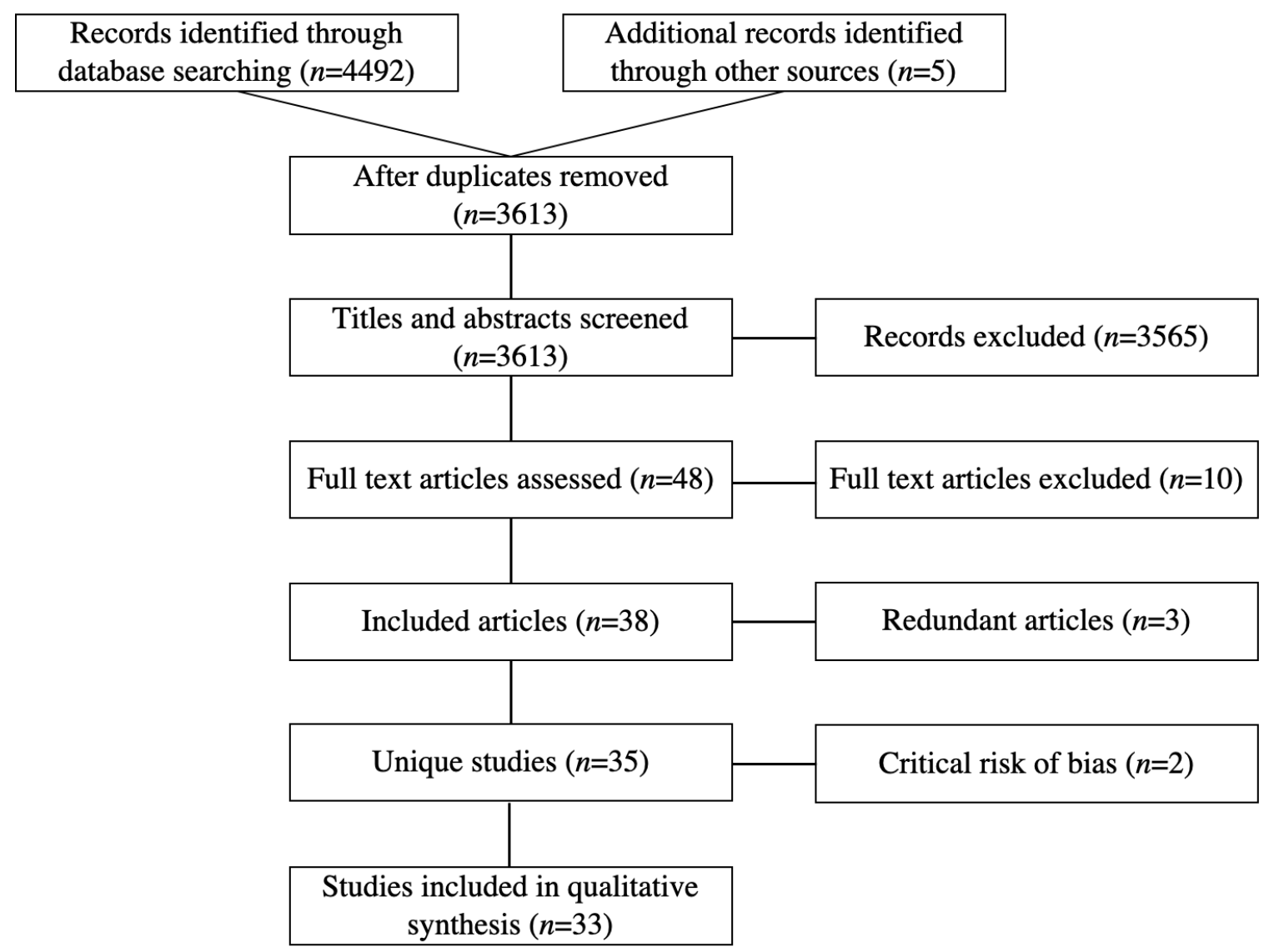

Figure 1. Modified preferred reporting items for systematic reviews and meta-analyses (PRISMA) flow diagram.

\subsection{Study Characteristics}

After full-text review, 35 studies were included [Figure 1]. This included eight RCTs [26-33], six CBA studies [34-39], four ITS studies [40-43], one historically controlled study [44], two prospective cohort studies [45,46], and fourteen uCBA studies [47-60]. Studies describing multiple similar interventions were considered as one study $[48,49,60]$. Of the 35 studies, 14 were from the USA [28,29,33,34,38,39,44,46,47,50,51,53,59,60], 5 from Italy [37,41-43,48], 2 from Australia [31,57], 2 from Denmark [26,52], and 1 each from Belgium [27], China [30], India [32], Cuba [35], Mexico [36], Germany [40], France [45], Nigeria [58], Finland [49], Egypt [54], Zimbabwe [55], and Canada [56].

All included studies were published between 2000-2018. Unless otherwise specified, all studies were published as a full paper. Characteristics of all included studies are summarized in Table 2. 
Table 2. Summary matrix of all included studies: tabulated data include information on industry, study design, participants, intervention, and outcomes.

\begin{tabular}{|c|c|c|c|c|c|c|}
\hline \multirow{3}{*}{$\begin{array}{l}\text { Agriculture, forestry, } \\
\text { fishing and hunting. }\end{array}$} & Study ID & Type of Study & Participants & Timeframe & $\begin{array}{l}\text { Intervention(s) and Control } \\
\text { (If Applicable) }\end{array}$ & Outcomes \\
\hline & Rasmussen et al., 2003 [26] & $\begin{array}{c}\mathrm{RCT} \text { randomized at the farm } \\
\text { level }\end{array}$ & $\begin{array}{c}\text { Farmers in Denmark } \\
\text { Intervention: } 99 \text { farms, } n=498 \\
\text { Control: } 102 \text { farms, } n=492\end{array}$ & $\begin{array}{l}\text { Duration: November } 1995 \\
\text { to July } 1997 \\
\text { Extended follow up: N/A }\end{array}$ & $\begin{array}{l}\text { Safety audits with feedback, } \\
\text { and a 1-day safety course } \\
\text { featuring didactic and } \\
\text { participatory education; } \\
\text { controls received no safety } \\
\text { course }\end{array}$ & $\begin{array}{l}\text { No significant effect in } \\
\text { preventing injuries compared to } \\
\text { control, even when adjusting for } \\
\text { seasonal variation-30\% } \\
\text { reduction in injury rate } \mathbf{9 5 \%} \text { CI: } \\
\mathbf{2 1 \%} \text { increase-60\% reduction) }\end{array}$ \\
\hline & Rautiainen et al., 2004 [29] & $\begin{array}{l}\text { Matched pairs cluster RCT, } \\
\text { randomized at the farm level }\end{array}$ & $\begin{array}{l}\text { Farmers in the USA } \\
\text { Intervention: } n=152 \text { farms } \\
\text { Control: } n=164 \text { farms }\end{array}$ & $\begin{array}{l}\text { Duration: } 1998 \text { to } 2003 \\
\text { Extended follow up: N/A }\end{array}$ & $\begin{array}{l}\text { Annual clinical screening, } \\
\text { access to didactic materials, } \\
\text { and a monetary incentive for } \\
\text { farmers who received a } \\
\text { safety audit score; controls } \\
\text { received no intervention }\end{array}$ & $\begin{array}{l}\text { No significant effect in } \\
\text { preventing injuries compared to } \\
\text { control, even when adjusting for } \\
\text { confounding factors-rate ratio } \\
\text { of injury rates (intervention vs. } \\
\text { control) was } \mathbf{1 . 0 8} \text { (96\% CI: } \\
\mathbf{0 . 8 6 - 1 . 3 7 )}\end{array}$ \\
\hline $\begin{array}{l}\text { Arts, Entertainment, } \\
\quad \& \text { Recreation }\end{array}$ & Geller et al., 2001 [33] & $\begin{array}{l}\text { Matched-pairs cluster RCT, } \\
\text { randomized at the pool level }\end{array}$ & $\begin{array}{l}\text { Pool staff in the USA } \\
\text { Intervention: } n=142 \\
\text { Control: } n=78\end{array}$ & $\begin{array}{l}\text { Duration: summer } 1999 \\
\text { Extended follow up: N/A }\end{array}$ & $\begin{array}{l}\text { Didactic education on } \\
\text { sunburns and skin cancer, } \\
\text { plus engineering and } \\
\text { personal protective } \\
\text { equipment support; the } \\
\text { control group received } \\
\text { placebo intervention on child } \\
\text { injury prevention. }\end{array}$ & $\begin{array}{l}\text { Significant effect in preventing } \\
\text { sunburns compared to } \\
\text { control-1.42 was the average } \\
\text { number of sunburns among } \\
\text { intervention participants, and } \\
\mathbf{2 . 0 7} \text { among control participants } \\
(p<0.05)\end{array}$ \\
\hline \multirow{4}{*}{ Construction } & Bena et al., $2009[41]^{1}$ & Interrupted time series & $\begin{array}{c}\text { Construction workers in Italy } \\
\text { Basic modules: } n=2320 \text { or } 2375 \\
\text { worker jobs } \\
\text { Specific modules: } n=1158 \text { or } \\
1164 \text { worker jobs }\end{array}$ & $\begin{array}{l}\text { Duration: July } 2002 \text { to } \\
\text { December } 2005 \\
\text { Extended follow up: N/A }\end{array}$ & $\begin{array}{l}\text { Didactic module-based } \\
\text { education, basic modules for } \\
\text { all workers and specific } \\
\text { modules for specific jobs }\end{array}$ & $\begin{array}{l}\text { No significant effect in } \\
\text { preventing injuries-estimated } \\
\mathbf{6 \%} \text { decrease in the frequency of } \\
\text { injuries per } 10,000 \text { days of } \\
\text { exposure }(p=0.08)\end{array}$ \\
\hline & $\begin{array}{l}\text { Kidd et al., } 2007 \text { (grant } \\
\text { report) [39] }\end{array}$ & Controlled before and after & $\begin{array}{c}\text { Construction workers in the USA } \\
\text { Intervention: } n=260 \\
\text { Control: } n=95\end{array}$ & $\begin{array}{l}\text { Duration: } 1995 \text { to } 1999 \\
\text { Extended follow up: N/A }\end{array}$ & $\begin{array}{l}\text { Participatory training for fall } \\
\text { prevention and back injury } \\
\text { prevention }\end{array}$ & $\begin{array}{l}\text { No significant differences in the } \\
\text { number or cost of injury claims } \\
\text { compared to control }\end{array}$ \\
\hline & Schoenfisch et al., 2017 [46] & Prospective cohort & $\begin{array}{c}\text { Carpenters in the USA } \\
\text { Exposed: } n=377 \text { with OSHA-10 } \\
\text { training, } n=76 \text { with OSHA-30 } \\
\text { training } \\
\text { Unexposed: } n=17,106\end{array}$ & $\begin{array}{l}\text { Duration: } 2000 \text { to } 2008 \\
\text { Extended follow up: N/A }\end{array}$ & $\begin{array}{l}\text { The Occupational Safety and } \\
\text { Health Administration } \\
\text { (OSHA) offered 10- and 30-h } \\
\text { didactic injury prevention } \\
\text { courses }\end{array}$ & $\begin{array}{l}\text { No significant effect in } \\
\text { preventing injuries - the rate } \\
\text { ratio of injuries in trained vs. } \\
\text { untrained workers was } 0.87 \text { ( } 95 \% \\
\text { CI: 0.72-1.06) }\end{array}$ \\
\hline & Evanoff et al., 2016 [50] & Uncontrolled Before and After & Carpenters in the USA, $n=2291$ & $\begin{array}{l}\text { Duration: } 2007 \text { to } 2009 \\
\text { Extended follow up: N/A }\end{array}$ & $\begin{array}{l}\text { Didactic and participatory } \\
\text { education on fall prevention }\end{array}$ & $\begin{array}{l}\text { No significant effect in } \\
\text { preventing injuries, once } \\
\text { covariates are accounted } \\
\text { for-incidence rate of falls (95\% } \\
\text { CI) pre-intervention: 18.3 } \\
\text { (15.5-21.1) vs. } \\
\text { post-intervention: } 14 \text { (11.7-16.2) }\end{array}$ \\
\hline
\end{tabular}


Table 2. Cont

\begin{tabular}{|c|c|c|c|c|c|c|}
\hline & Study ID & Type of Study & Participants & Timeframe & $\begin{array}{c}\text { Intervention(s) and Control } \\
\text { (If Applicable) }\end{array}$ & Outcomes \\
\hline & Darragh et al., 2004 [51] & Uncontrolled Before and After & $\begin{array}{l}\text { Construction workers in the USA } \\
n=97 \text { companies }\end{array}$ & $\begin{array}{l}\text { Duration: } 1997 \text { to } 1998 \\
\text { Extended follow up: N/A }\end{array}$ & $\begin{array}{l}\text { Three-hour safety training } \\
\text { and orientation, plus access } \\
\text { to optional 10-h OSHA } \\
\text { training courses }\end{array}$ & $\begin{array}{l}\text { No significant effect in } \\
\text { preventing injuries-the risk } \\
\text { ratio before and after } \\
\text { intervention was } 0.97 \text { (95\% CI: } \\
0.5-2.0)\end{array}$ \\
\hline & Johnson \& Ruppe 2002 [59] & Uncontrolled before and after & $\begin{array}{l}\text { Construction workers in the } \\
\text { USA, } n=55\end{array}$ & $\begin{array}{l}\text { Duration: } 1998 \text { to } 1999 \\
\text { Extended follow up: N/A }\end{array}$ & $\begin{array}{l}\text { Didactic education, plus } \\
\text { stress management and } \\
\text { spirituality sessions }\end{array}$ & $\begin{array}{l}\text { Although injury rates decreased, } \\
\text { statistical significance was not } \\
\text { stated }\end{array}$ \\
\hline & Spangenberg et al., 2002 [52] & Uncontrolled before and after & $\begin{array}{l}\text { Construction workers in } \\
\text { Denmark, sample size not stated, } \\
\text { but estimated } 6.8 \text { million } \\
\text { person-hours worked }\end{array}$ & $\begin{array}{l}\text { Duration: } 1996 \text { to } 1998 \\
\text { Extended follow up: N/A }\end{array}$ & $\begin{array}{l}\text { Didactic education, } \\
\text { safety-based monetary } \\
\text { incentives, and a social } \\
\text { marketing campaign }\end{array}$ & $\begin{array}{l}\text { Significant effect in preventing } \\
\text { injuries, once concurrent changes } \\
\text { in construction intensity are } \\
\text { accounted for-25\% reduction } \\
\text { in injuries }(95 \% \text { CI: } 1-43 \%)\end{array}$ \\
\hline & Williams et al., 2010 [53] & Uncontrolled before and after & $\begin{array}{l}\text { Construction workers in the USA } \\
\text { (all Latino males), } n=313\end{array}$ & $\begin{array}{l}\text { Duration: } 1 \text { day } \\
\text { Extended follow up: } 2-6 \\
\text { months }\end{array}$ & $\begin{array}{l}\text { Didactic education on topics } \\
\text { pertinent to Latino } \\
\text { immigrant workers }\end{array}$ & $\begin{array}{l}\text { Although injury rates decreased } \\
\text { by } 24.0 \% \text {, statistical significance } \\
\text { was not stated }\end{array}$ \\
\hline Education Services & Vercruysse et al., 2016 [27] & $\begin{array}{l}\text { Cluster RCT, randomized at } \\
\text { the school level }\end{array}$ & $\begin{array}{c}\text { Physical education teachers in } \\
\text { Belgium } \\
\text { Intervention: } n=29 \text { teachers } \\
\text { from } 19 \text { schools } \\
\text { Control: } n=26 \text { teachers from } 20 \\
\text { schools }\end{array}$ & $\begin{array}{l}\text { Duration: September } 2013 \\
\text { to June } 2014 \\
\text { Extended follow up: N/A }\end{array}$ & $\begin{array}{l}\text { Didactic and participatory } \\
\text { education, access to didactic } \\
\text { videos; controls did not } \\
\text { receive any intervention }\end{array}$ & $\begin{array}{l}\text { No significant effect in } \\
\text { preventing injuries compared to } \\
\text { control-0.20 (95\% CI: } 0.06-0.61) \\
\text { work injuries per } 1000 \mathrm{~h} \text { in the } \\
\text { intervention group; } \mathbf{0 . 5 4} \\
(\mathbf{0 . 2 4 - 1 . 1 8 )} \text { work injuries per } \\
1000 \mathrm{~h} \text { in the control group }\end{array}$ \\
\hline $\begin{array}{l}\text { Health Care \& Social } \\
\text { Assistance }\end{array}$ & Koblesky 2017 (abstract) [47] & Uncontrolled before and after & $\begin{array}{l}\text { Blood center employees in the } \\
\text { USA, } 2010 \text { to } 2014 \\
\text { Sample size not stated }\end{array}$ & $\begin{array}{l}\text { Duration: } 2011 \text { to } 2014 \\
\text { Extended follow up: N/A }\end{array}$ & $\begin{array}{l}\text { Didactic education and social } \\
\text { marketing, plus } \\
\text { administrative changes }\end{array}$ & $\begin{array}{l}\text { Although the number of injuries, } \\
\text { workers' compensation claims, } \\
\text { and days away from work } \\
\text { decreased, statistical significance } \\
\text { was not stated }\end{array}$ \\
\hline \multirow{2}{*}{ Manufacturing } & Yu et al., 2017 [30] & Paired cluster RCT & $\begin{array}{l}\text { Factory workers in China } \\
\text { Intervention: } n=966 \text { from } 30 \\
\text { experimental factories } \\
\text { Control 1: } n=966 \text { from the same } \\
30 \text { experimental factories } \\
\text { Control } 2: n=1706 \text { from } 30 \\
\text { control factories }\end{array}$ & $\begin{array}{l}\text { Duration: June } 2008 \text { to } \\
\text { November } 2009 \\
\text { Extended follow up: } 12 \\
\text { months }\end{array}$ & $\begin{array}{l}\text { Participatory education } \\
\text { featuring a workplace } \\
\text { inspection exercise followed } \\
\text { by discussion on } \\
\text { implementable actions; } \\
\text { controls received didactic } \\
\text { education only }\end{array}$ & $\begin{array}{l}\text { Significant effect in preventing } \\
\text { injuries compared to control and } \\
\text { baseline. Compared to the } \\
\text { experimental group, the odds } \\
\text { ratio (95\% CI) was 1.78 } \\
\text { (1.04-3.04) for experimental } \\
\text { factory controls, and } 1.77 \\
\text { (1.13-2.79) for control factory } \\
\text { controls }\end{array}$ \\
\hline & Morgan et al., 2012 [31] & Individually randomized RCT & $\begin{array}{c}\text { Overweight/obese (BMI 25-40) } \\
\text { male aluminum workers in } \\
\text { Australia } \\
\text { Intervention: } n=65 \\
\text { Control: } n=45\end{array}$ & $\begin{array}{l}\text { Duration: October } 2009 \text { to } \\
\text { March } 2010 \\
\text { Extended follow up: } 12 \\
\text { months }\end{array}$ & $\begin{array}{l}\text { Didactic education, lifestyle } \\
\text { feedback, free pedometers, } \\
\text { and a monetary incentive to } \\
\text { lose weight (although the } \\
\text { stated goal was obesity } \\
\text { reduction, injury prevention } \\
\text { was an intended effect of } \\
\text { intervention); controls were } \\
\text { put on a waitlist }\end{array}$ & $\begin{array}{l}\text { Significant effect in preventing } \\
\text { injuries compared to } \\
\text { control-the mean difference } \\
\text { between groups was } 0.3 \text { fewer } \\
\text { injuries per person for the } \\
\text { intervention group }(95 \% \text { CI: } \\
0.0-0.6)\end{array}$ \\
\hline
\end{tabular}


Table 2. Cont

\begin{tabular}{|c|c|c|c|c|c|}
\hline Study ID & Type of Study & Participants & Timeframe & $\begin{array}{l}\text { Intervention(s) and Control } \\
\text { (If Applicable) }\end{array}$ & Outcomes \\
\hline Hermann et al., $2010[36]^{1}$ & Controlled before and after & $\begin{array}{l}\text { Automobile plant workers in } \\
\text { Mexico } \\
\text { Intervention: one plant } \\
\text { (workforce 873-1350) } \\
\text { Control: two plants (workforces } \\
\text { 2990-3800 and 1291-1369 } \\
\text { respectively) }\end{array}$ & $\begin{array}{l}\text { Duration: January } 1997 \text { to } \\
\quad \text { January } 2004 \\
\text { Extended follow up: N/A }\end{array}$ & $\begin{array}{l}\text { Didactic education, a social } \\
\text { marketing campaign, and } \\
\text { behavioral feedback, plus } \\
\text { administrative changes; } \\
\text { controls received a basic } \\
\text { safety campaign }\end{array}$ & $\begin{array}{l}\text { Significant effect in preventing } \\
\text { injuries compared to } \\
\text { baseline- } \mathbf{9 2} \% \text { percent decrease } \\
\text { of medical plus lost-time cases in } \\
\text { the experimental plant, } 3 \% \text { in } \\
\text { control plant A, and } 6 \% \text { in } \\
\text { control plant B }\end{array}$ \\
\hline Mancini et al., 2005 [37] & Controlled before and after & $\begin{array}{l}\text { Factory workers in Italy } \\
\text { Intervention: } n=237 \text { metal-ware } \\
\text { factories (workforce not stated) } \\
\text { Control: construction and } \\
\text { wood/ceramics industries } \\
\text { (workforce not stated) }\end{array}$ & $\begin{array}{l}\text { Duration: December } 1991 \\
\text { to June } 1992 \\
\text { Extended follow up: } 11 \\
\text { years }\end{array}$ & $\begin{array}{l}\text { Didactic education and a } \\
\text { social marketing campaign; } \\
\text { controls received no } \\
\text { intervention }\end{array}$ & $\begin{array}{l}\text { Significant effect in preventing } \\
\text { eye injuries compared to } \\
\text { control-post-intervention } \\
\text { incident rate ratios }(95 \% \mathrm{CI}) \\
\text { were } 0.77(\mathbf{0 . 6 1 - 0 . 9 7 )} \text { after 1-4 } \\
\text { years, } 0.63(0.50-0.79) \text { after 5-8 } \\
\text { years, and } 0.58(0.43-0.77) \text { after } \\
\text { 9-11 years }\end{array}$ \\
\hline Yeow \& Goomas 2014 [38] & $\begin{array}{l}\text { Controlled before and after } \\
\text { study }\end{array}$ & $\begin{array}{l}\text { Fluid plant workers in the USA } \\
\text { Intervention: one fluid } \\
\text { manufacturing plant, } n=362 \\
\text { Control: one fluid } \\
\text { manufacturing plant, } n=338\end{array}$ & $\begin{array}{l}\text { Duration: } 26 \text { months } \\
\text { Extended follow up: N/A }\end{array}$ & $\begin{array}{l}\text { A safety-based monetary } \\
\text { incentive program, } \\
\text { peer-based monitoring and } \\
\text { safety discussions; controls } \\
\text { received didactic lectures } \\
\text { only }\end{array}$ & $\begin{array}{l}\text { Significant effect in preventing } \\
\text { injuries compared to control } \\
\text { (48\% reduction after } 2 \text { years) } \\
\text { and baseline ( } 33 \% \text { reduction } \\
\text { after } 2 \text { years) }\end{array}$ \\
\hline Porru et al., 2011 [42] & Interrupted Time Series & $\begin{array}{c}\text { Foundry workers in Italy } \\
\text { One ferrous foundry }(n=230 \\
\text { approximately) and one } \\
\text { non-ferrous foundry }(n=50 \\
\text { approximately) }\end{array}$ & $\begin{array}{l}\text { Duration: 2000-2002 } \\
\text { Extended follow up: } 7 \\
\text { years }\end{array}$ & $\begin{array}{l}\text { Safety discussions, didactic } \\
\text { and participatory education, } \\
\text { technical and organizational } \\
\text { support, and health } \\
\text { surveillance }\end{array}$ & $\begin{array}{l}\text { Significant effect in preventing } \\
\text { injuries in the short, medium, } \\
\text { and long term for foundry A, but } \\
\text { only in the long term for foundry } \\
\text { B }\end{array}$ \\
\hline Porru et al., 2017 [43] & Interrupted time series & $\begin{array}{c}\text { Foundry workers in Italy } \\
22 \text { ferrous (total } n=2750 \\
\text { workers) and } 7 \text { non-ferrous } \\
\text { foundries (total } n=710 \text { workers) }\end{array}$ & $\begin{array}{l}\text { Duration: } 2008 \text { to } 2013 \\
\text { Extended follow up: N/A }\end{array}$ & $\begin{array}{l}\text { Improved formalization and } \\
\text { dissemination of safety } \\
\text { instructions, didactic } \\
\text { education, safety audits and } \\
\text { administrative support, and } \\
\text { health surveillance }\end{array}$ & $\begin{array}{l}\text { Only significant } 26 \% \text { (95\% CI: } \\
5-43 \% \text { ) reduction in incidence } \\
\text { rate (per worker) but not } \\
\text { frequency rate (per hour) in } \\
\text { ferrous foundries; no significant } \\
\text { differences found in non-ferrous } \\
\text { foundries }\end{array}$ \\
\hline $\begin{array}{l}\text { Borger et al., } 2011 \text { (abstract) } \\
\text { [40] }\end{array}$ & Interrupted time series & $\begin{array}{l}\text { Glass factory workers in } \\
\text { Germany } \\
10 \text { glass factories, } n=860\end{array}$ & $\begin{array}{l}\text { Duration: } 2002 \text { to } 2003 \\
\text { Extended follow up: } 6 \\
\text { years }\end{array}$ & $\begin{array}{l}\text { Training on job-specific safety } \\
\text { behaviors and risk } \\
\text { management }\end{array}$ & $\begin{array}{l}\text { Significant effect in preventing } \\
\text { injuries-ITS reveals a } 37 \% \\
\text { decrease that can be attributed to } \\
\text { intervention }\end{array}$ \\
\hline Shouman et al., 2002 [54] & Uncontrolled before and after & $\begin{array}{l}\text { Glass factory workers in Egypt, } n \\
=1229\end{array}$ & $\begin{array}{c}\text { Duration: } 2000 \text { calendar } \\
\text { year } \\
\text { Extended follow up: N/A }\end{array}$ & $\begin{array}{l}\text { Didactic education, social } \\
\text { marketing, a safety-based } \\
\text { monetary incentive, greater } \\
\text { availability of PPE, and } \\
\text { administrative support }\end{array}$ & $\begin{array}{l}\text { Significant effect in preventing } \\
\text { injuries-24\% reduction in both } \\
\text { incidence rate (per worker) and } \\
\text { frequency rate (per hour) }\end{array}$ \\
\hline
\end{tabular}


Table 2. Cont.

\begin{tabular}{|c|c|c|c|c|c|c|}
\hline & Study ID & Type of Study & Participants & Timeframe & $\begin{array}{l}\text { Intervention(s) and Control } \\
\text { (If Applicable) }\end{array}$ & Outcomes \\
\hline & Nunu et al., 2018 [55] & Uncontrolled before and after & $\begin{array}{l}\text { Cement manufacturing workers } \\
\text { in Zimbabwe, } n=244\end{array}$ & $\begin{array}{l}\text { Duration: } 2007 \text { to } 2011 \\
\text { Extended follow up: N/A }\end{array}$ & $\begin{array}{l}\text { Peer-based behavioral } \\
\text { monitoring and } \\
\text { reinforcement; rewards for } \\
\text { safe behavior and } \\
\text { reorientation for unsafe } \\
\text { behavior }\end{array}$ & $\begin{array}{l}\text { Significant effect in preventing } \\
\text { injuries- } 37 \% \text { reduction in the } \\
\text { number of injuries }\end{array}$ \\
\hline & Day 2002 (thesis) [56] & Uncontrolled before and after & $\begin{array}{l}\text { Workers at a pulp and paper mill } \\
\text { in Canada, } n=190\end{array}$ & $\begin{array}{l}\text { Duration: February to } \\
\text { March 2002 } \\
\text { Extended follow up: } 2 \\
\text { months } \\
\end{array}$ & $\begin{array}{l}\text { One-day safety leadership } \\
\text { course, all workers were } \\
\text { welcome to attend }\end{array}$ & $\begin{array}{l}\text { Although medical and first-aid } \\
\text { incidents increased, statistical } \\
\text { significance was not stated }\end{array}$ \\
\hline & $\begin{array}{l}\text { Gatti et al., } 2013 \text { (abstract) } \\
\text { [48] }\end{array}$ & Uncontrolled before and after & $\begin{array}{l}\text { Workers in } 2 \text { factories in Italy, } \\
\text { sample sizes not stated }\end{array}$ & $\begin{array}{l}\text { Duration: both studies are } \\
2010 \text { to } 2012 \\
\text { Extended follow up: N/A }\end{array}$ & $\begin{array}{l}\text { Behavioral feedback, } \\
\text { reinforcement, and problem } \\
\text { solving }\end{array}$ & $\begin{array}{l}\text { Although injury rates decreased } \\
\text { by } 52 \% \text { in the first factory and } \\
\mathbf{6 8 \%} \text { in the second factory, } \\
\text { statistical significance was not } \\
\text { stated in either }\end{array}$ \\
\hline \multirow{3}{*}{$\begin{array}{l}\text { Mining, Quarrying, } \\
\text { and Oil and Gas } \\
\text { Extraction }\end{array}$} & Adams et al., 2013 [32] & $\begin{array}{l}\text { Cluster-randomized RCT at the } \\
\text { quarry level }\end{array}$ & $\begin{array}{l}\text { Stone quarry workers in India } \\
\text { Intervention: } n=103 \text { from three } \\
\text { experimental quarries } \\
\text { Control: } n=101 \text { from three } \\
\text { control quarries }\end{array}$ & $\begin{array}{l}\text { Duration: September } 2006 \\
\text { to March } 2007 \\
\text { Extended follow up: N/A }\end{array}$ & $\begin{array}{l}\text { Eleven sessions of didactic } \\
\text { education, ocial marketing, } \\
\text { group motivational sessions, } \\
\text { and individual counselling; } \\
\text { controls received one session } \\
\text { of standard didactic } \\
\text { education }\end{array}$ & $\begin{array}{l}\text { Significant effect in preventing } \\
\text { injuries compared to } \\
\text { baseline-12\% reduction } \mathbf{9 5 \%} \\
\text { CI: } \mathbf{3 - 2 1} \% \text { ) }\end{array}$ \\
\hline & Hagge et al., 2016 [34] & Controlled before and after & $\begin{array}{c}\text { Miners in the USA } \\
\text { Intervention: } n=\text { approximately } \\
400 \\
\text { Control: industry standard }\end{array}$ & $\begin{array}{l}\text { Duration: } 2007 \text { to } 2014 \\
\text { Extended follow up: N/A }\end{array}$ & $\begin{array}{l}\text { Peer safety observation and } \\
\text { feedback, and creation of a } \\
\text { new safety plan, plus } \\
\text { safety-oriented } \\
\text { administrative changes }\end{array}$ & $\begin{array}{l}\text { Although injury rates decreased } \\
\text { by } 50 \% \text {, statistical significance } \\
\text { was not stated }\end{array}$ \\
\hline & $\begin{array}{l}\text { Kowalski-Trakofler \& Barrett } \\
2016 \text { [60] }\end{array}$ & Uncontrolled before and after & $\begin{array}{l}\text { Miners in the USA } \\
\text { Study B: } 4 \text { mines with > } 2300 \\
\text { workers total; Study C: } 1 \text { mine } \\
\text { with } 550 \text { workers (Study A not } \\
\text { included due to lack of injury } \\
\text { outcomes) }\end{array}$ & $\begin{array}{l}\text { Duration: } 1995 \text { to } 1996 \text { for } \\
\text { Study B; } 1995 \text { for Study C } \\
\text { Extended follow up: } 12 \\
\text { months for Study C }\end{array}$ & $\begin{array}{l}\text { Degraded images were used } \\
\text { instead of highlighted images } \\
\text { during safety training }\end{array}$ & $\begin{array}{l}\text { Significant effect in preventing } \\
\text { injuries in study B }(\mathbf{9 . 0 6 \%} \\
\text { decrease in the first year and a } \\
\text { further } 29.94 \% \text { in the second } \\
\text { year); although injury rates } \\
\text { decreased by } 27.1 \% \text { in study C, } \\
\text { significance was not stated }\end{array}$ \\
\hline \multirow[t]{2}{*}{ Public Administration } & Sullivan et al., 2017 [28] & $\begin{array}{l}\text { Matched-pairs cluster RCT, } \\
\text { randomized at the station level }\end{array}$ & $\begin{array}{l}\text { Firefighters in the USA } \\
\text { Intervention: } 16 \text { stations, } n=601 \\
\text { Control: } 16 \text { stations, } n=588\end{array}$ & $\begin{array}{l}\text { Duration: last } 2 \text { weeks of } \\
\text { August } 2009 \\
\text { Extended follow up: } 54 \\
\text { weeks }\end{array}$ & $\begin{array}{l}\text { Didactic education on sleep } \\
\text { health (although the stated } \\
\text { goal was to improve sleep } \\
\text { health, injury prevention was } \\
\text { an intended effect of } \\
\text { intervention); controls did } \\
\text { not receive intervention }\end{array}$ & $\begin{array}{l}\text { Significant effect in reducing the } \\
\text { number of injury and disability } \\
\text { days (1.4 per worker in the } \\
\text { intervention group vs. 2.6 per } \\
\text { worker in the control group), } \\
\text { but not the number of injuries } \\
\text { and motor vehicle crashes }\end{array}$ \\
\hline & Knapik et al., 2004 [44] & Historically controlled & $\begin{array}{l}\text { Soldiers in the USA } \\
\text { Intervention: } n=1283 \text { (1122 men } \\
\text { and } 161 \text { women) } \\
\text { Historical control: } n=2559 \text { (2303 } \\
\text { men and } 256 \text { women) }\end{array}$ & $\begin{array}{l}\text { Duration: } 36 \text { weeks } \\
\text { Extended follow up: N/A }\end{array}$ & $\begin{array}{l}\text { Modified physical training } \\
\text { and didactic education, plus } \\
\text { administrative injury } \\
\text { surveillance support }\end{array}$ & $\begin{array}{l}\text { Significant effect in preventing } \\
\text { injuries for men only-adjusted } \\
\text { risk ratio of control vs. } \\
\text { intervention }(95 \% \text { CI) was } \mathbf{1 . 5 0} \\
\mathbf{( 1 . 0 6 - 2 . 1 2 )} \text { for men and } \mathbf{1 . 3 7} \\
\mathbf{( 0 . 5 7 - 3 . 2 9 ) ~ f o r ~ w o m e n ~}\end{array}$ \\
\hline
\end{tabular}


Table 2. Cont

\begin{tabular}{|c|c|c|c|c|c|c|}
\hline \multirow{3}{*}{$\begin{array}{l}\text { Transportation and } \\
\text { Warehousing }\end{array}$} & Study ID & Type of Study & Participants & Timeframe & $\begin{array}{l}\text { Intervention(s) and Control } \\
\text { (If Applicable) }\end{array}$ & Outcomes \\
\hline & Robaina et al., 2010 [35] & Controlled before and after & $\begin{array}{c}\text { Stevedores in Cuba } \\
\text { Intervention: } n=185 \text { (from one } \\
\text { terminal) } \\
\text { Control: } n=105 \text { (from another } \\
\text { terminal) }\end{array}$ & $\begin{array}{l}\text { Duration: January } 2004 \text { to } \\
\text { April } 2005 \\
\text { Extended follow up: until } \\
\text { end of } 2006\end{array}$ & $\begin{array}{l}\text { Group safety discussions, } \\
\text { didactic and participatory } \\
\text { education for workers and } \\
\text { supervisors; controls } \\
\text { received no intervention. } \\
\end{array}$ & $\begin{array}{l}\text { Significant effect in preventing } \\
\text { injuries compared to control } \\
\text { ( } 58.8 \% \text { of injuries prevented) } \\
\text { and baseline ( } 2.8 \text { fewer injuries } \\
\text { per } 100 \text { person-years) }\end{array}$ \\
\hline & $\begin{array}{l}\text { Howard et al., } 2009 \text { (abstract) } \\
\text { [57] }\end{array}$ & Uncontrolled before and after & $\begin{array}{l}\text { Road transport drivers, } \\
\text { Australia, } n=800\end{array}$ & $\begin{array}{l}\text { Duration: } 3 \text { years } \\
\text { Extended follow up: } 12 \\
\text { months }\end{array}$ & $\begin{array}{l}\text { Sleep health education and } \\
\text { individual health screening, } \\
\text { (although the stated goal was } \\
\text { to improve sleep health, } \\
\text { injury prevention was an } \\
\text { intended effect of } \\
\text { intervention) }\end{array}$ & $\begin{array}{l}\text { Significant effect in preventing } \\
\text { injuries-lost time injuries per } \\
100 \text { drivers were reduced from } \\
\mathbf{1 7 . 1} \text { to } \mathbf{1 4 . 2}\end{array}$ \\
\hline \multirow[t]{2}{*}{ Utilities } & Salminen 2008 [49] & Uncontrolled before and after & $\begin{array}{c}\text { Electricians in Finland, } 1998 \text { to } \\
\text { 2005 } \\
\text { Study 1: } n=172 \\
\text { Study 2: } n=179\end{array}$ & $\begin{array}{l}\text { Duration: } 2001 \text { to } 2002 \text { for } \\
\text { Study 1; } 2001 \text { for Study } 2 \\
\text { Extended follow up: } 3 \\
\text { years for both studies }\end{array}$ & $\begin{array}{l}\text { Study 1: group safety } \\
\text { discussion followed by } \\
\text { collaborative decision on } \\
\text { solutions } \\
\text { Study 2: Didactic and } \\
\text { participatory education on } \\
\text { driving safety }\end{array}$ & $\begin{array}{l}\text { In Study } 1 \text {, although } \\
\text { work-related traffic incidents } \\
\text { decreased by } 72.2 \% \text {, other } \\
\text { occupational injuries increased } \\
\text { by } 15.1 \% \text {. The proportion of } \\
\text { traffic-related incidents } \\
\text { decreased significantly. In Study } \\
2 \text {, although the rate of injuries } \\
\text { increased by } 50 \% \text {, statistical } \\
\text { significance was not stated }\end{array}$ \\
\hline & Badmos 2018 (abstract) [58] & Uncontrolled before and after & $\begin{array}{l}\text { Electricity distribution company } \\
\text { employees in Nigeria, (sample } \\
\text { size not stated) }\end{array}$ & $\begin{array}{l}\text { Duration: } 2015 \text { to } 2017 \\
\text { Extended follow up: N/A }\end{array}$ & $\begin{array}{l}\text { Safety counselling and } \\
\text { videos, safety huddles, and } \\
\text { hazard identification } \\
\text { competitions, plus } \\
\text { administrative changes }\end{array}$ & $\begin{array}{l}\text { Although injury rates decreased } \\
\text { by } 40 \% \text { among staff, statistical } \\
\text { significance was not stated. }\end{array}$ \\
\hline Mixed Industries & Boini et al., 2017 [45] & Prospective cohort & $\begin{array}{l}\text { Young workers in France, } \\
\text { 2009-2014 } \\
\text { Exposed: students who received } \\
\text { occupational safety and health } \\
\text { (OSH) training in school, } n=687 \\
\text { Unexposed: students who did } \\
\text { not receive training, } n=68\end{array}$ & $\begin{array}{l}\text { Duration: variable } \\
\text { Extended follow up: } 2 \\
\text { years }\end{array}$ & $\begin{array}{l}\text { Didactic education (varied } \\
\text { based on type of diploma) }\end{array}$ & $\begin{array}{l}\text { Significant effect in preventing } \\
\text { injuries - the incident rate ratio } \\
\text { of exposed to unexposed was } \\
0.51 \text { ( } 95 \% \text { CI: } 0.00-0.98 \text { ). }\end{array}$ \\
\hline
\end{tabular}

${ }^{1}$ Not included in narrative synthesis due to critical risk of bias. 
There were 4 RCTs, 4 CBAs, 2 ITSs, 1 historically controlled study, 1 prospective cohort, and 5 uCBAs which demonstrated a significant reduction of injuries [30-33,35-38,40,42,44,45,52,54,55,57,60]. However, 4 RCTs, 2 CBAs, 2 ITSs, 1 prospective cohort, and 9 uCBAs produced equivocal or non-significant results $[26-29,34,39,41,43,46-51,53,56,58,59]$. Sources of funding and conflicts of interest for included studies are shown in Appendix C.

\subsection{Risk of Bias in Included Studies}

Of the eight RCT studies, five were judged to be at moderate risk of bias using the RoB 2 tool, and three were at high risk of bias (Figure 2).

\begin{tabular}{|c|c|c|c|c|c|c|}
\hline Study ID & 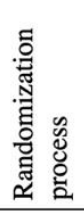 & 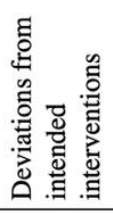 & 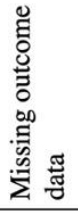 & 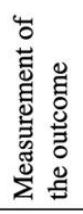 & 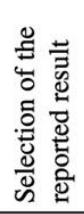 & 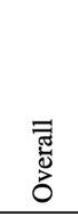 \\
\hline Adams et al 2013 & Low & Low & Low & Low & Some & Some \\
\hline Geller at al 2001 & High & High & Low & High & High & High \\
\hline Morgan et al 2012 & Low & Some & Low & Low & Some & Some \\
\hline Rasmussen et al 2003 & Some & Some & Low & Low & Some & Some \\
\hline Rautiainen et al 2004 & Some & Some & High & Low & Some & High \\
\hline Sullivan et al 2016 & Some & Some & Low & Low & Some & Some \\
\hline Vercruysse et al 2016 & Low & High & Low & Low & Some & High \\
\hline Yu et al 2017 & Low & Low & Low & Low & Some & Some \\
\hline
\end{tabular}

Figure 2. Risk of bias in randomized controlled trial (RCT) studies-risk of bias was calculated using the risk of bias (RoB) 2 tool. The level of bias for each of the 5 domains are shown above for the 8 RCT studies.

Using the ROBINS-I tool, of the five CBA studies that were published as a full paper, four were at serious risk of bias and one was at critical risk of bias. Of the three ITS studies that were published as full papers, two were at moderate risk of bias, and one was at critical risk of bias. The historically controlled study and both of the prospective cohort studies were at serious risk of bias. The two studies with critical risk of bias will not be further discussed in the narrative synthesis $[36,41]$ (Figure 3).

As discussed previously, all $14 \mathrm{uCBAs}$ were automatically assigned a high risk of bias [24]. Risk of bias assessment was not attempted for the remaining abstract (ITS) and the grant report (CBA) due to insufficient information. 


\begin{tabular}{|c|c|c|c|c|c|c|c|c|}
\hline Study ID & 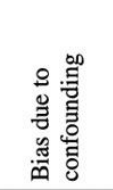 & 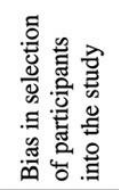 & 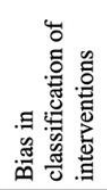 & 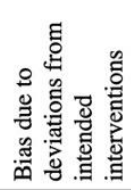 & 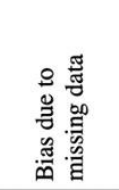 & 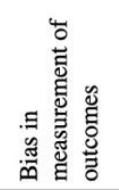 & 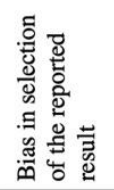 & 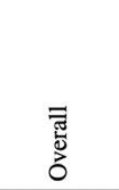 \\
\hline Bena et al 2009 & Serious & Critical & Critical & Low & Serious & Low & Moderate & Critical \\
\hline Boini et al 2017 & Serious & Low & Low & Low & Moderate & Low & Moderate & Serious \\
\hline Hagge et al 2016 & Serious & Unknown & Low & Low & Unknown & Serious & Moderate & Serious \\
\hline Hermann et al 2010 & Serious & Serious & Serious & Critical & Unknown & Low & Moderate & Critical \\
\hline Knapik et al 2004 & Serious & Low & Low & Low & Low & Low & Moderate & Serious \\
\hline Mancini et al 2005 & Serious & Low & Low & Low & Low & Low & Moderate & Serious \\
\hline Porru et al 2011 & Moderate & Unknown & Low & Low & Low & Low & Moderate & Moderate \\
\hline Porru et al 2017 & Moderate & Low & Low & Low & Low & Low & Moderate & Moderate \\
\hline Robaina et al 2010 & Serious & Low & Low & Low & Unknown & Unknown & Moderate & Serious \\
\hline Schoenfisch et al 2017 & Serious & Low & Low & Low & Low & Low & Moderate & Serious \\
\hline Yeow et al 2014 & Serious & Serious & Serious & Low & Low & Serious & Serious & Serious \\
\hline
\end{tabular}

Figure 3. Risk of bias in non-randomized controlled studies-risk of bias was calculated using the ROBINS-I tool. The level of bias for each of the 7 domains are shown above for the 11 non-randomized controlled studies. Note that the terminology used between RoB 2 and ROBINS-I is slightly different.

\section{Discussion}

Despite widespread use of education in occupational injury prevention programs, limited reviews exist on assessing injury outcomes in recent years. Hence, we aimed to narratively summarize the effects of educational interventions on occupational injury outcomes through this study. We systematically searched multiple databases and various sources of grey literature. A strength of our study is that we included grey literature, including abstracts, theses, and grant reports to avoid publication bias. However, a necessary trade-off was that their interventions and results were not always described in detail. Further, by considering a broad range of injuries across all industries, the results of this study could be more generalizable. To the best of our understanding, this is the first systematic review of its kind that is not limited to a particular industry or type of injury.

Our results reveal modest evidence that educational interventions have a protective effect against occupational injuries. However, the overall risk of bias was moderate to high among all included studies. In addition, the majority of all studies were performed in two industries, construction and manufacturing. Multifactorial and non-didactic educational interventions were generally more effective than didactic education. This is especially evident in the manufacturing sector, which employed the former strategies more frequently.

\subsection{Agriculture, Forestry, Fishing, and Hunting}

The agricultural sector experiences the highest rates of non-fatal injuries among all US industries [61]. However, neither of the RCTs included were able to demonstrate a significant reduction in injuries, despite utilizing multiple forms of education, such as didactic and interactive teaching, plus behavioral-based incentives [26,29]. Both studies were conducted in developed countries with a relatively high degree of legislative and engineering support. While this gives us some insight into the effect of education in developed countries, it limits our ability to extrapolate the findings to developing countries, where most of the world's farming population resides [62]. Our findings corroborate a meta-analysis by Rautiainen et al., who concluded that there was no evidence suggesting 
benefit from the use of educational interventions alone in the agricultural context, and that more high-quality studies, such as RCTs or ITSs, should be conducted for behavioral interventions [11].

\subsection{Arts, Entertainment, and Recreation}

Only one RCT was identified, targeting sunburn reduction among swimming pool staff [33]. This limits our ability to generalize the results to the rest of this sector. Nonetheless, this study is notable for using a placebo intervention in the control group, in which participants learned about child injury prevention. This strategy could help reduce the risk of bias associated with the impracticality of blinding participants in educational interventions.

\subsection{Construction}

Although a large number of studies were identified in construction, not all of them were methodologically sound. Our search revealed one CBA [39], one ITS which was removed from narrative synthesis due to critical risk of bias [41], one prospective cohort [46], and five uCBAs [50-53,59]. The overall results were not encouraging. Only one uCBA study found a statistically significant effect following an educational intervention [52]. Thus, there may be challenges in influencing the construction sector through purely educational means. This echoes a previous meta-analysis by van der Molen et al., which showed no strong evidence to suggest that safety campaigns alone have a protective effect on construction workers [10]. There may be a potential synergistic effect between educational and regulatory interventions, which may serve as a potential direction for future research $[63,64]$. It should also be noted that construction studies besides Spangenberg et al. [52], Evanoff et al. [50], and Kidd et al. [39] featured interventions that were almost exclusively didactic in nature. Interestingly, Spangenberg et al. was also the only study to find a significant effect, and Evanoff et al. found a significant effect before adjusting for covariates. This may indicate that non-didactic education is more effective, hence suggesting a direction for future research.

\subsection{Educational Services}

One RCT was identified [27], which did not find a significant benefit from educational intervention. Since the study was conducted in only one region in Belgium, risk of cross-contamination may have skewed the results. Moreover, since its results were analyzed on a per-protocol basis, it limits our ability to extrapolate results to the real world, where non-adherence may be prevalent.

\subsection{Health Care and Social Assistance}

Only one uCBA abstract was identified [47], which did not state statistical significance. There was insufficient information for conclusions to be drawn for this sector of the economy.

\subsection{Manufacturing}

Manufacturing had the largest number of included studies: two RCTs [30,31], three CBAs [36-38] (one of which was removed from narrative synthesis due to critical risk of bias [36]), three ITS studies $[40,42,43]$, and four uCBAs [48,54-56]. Of which, all of the RCT and CBA studies, one of the ITS studies [42], and two of the uCBA studies [54,55] showed significant injury reductions. Interestingly, one commonality among the majority of effective interventions in manufacturing was that they employed either multifactorial approaches or educational methods that were not purely didactic $[30,31,38,42,54,55]$. Despite the possibility of cross-contamination favoring the controls, these interventions still resulted in significantly positive results, which may indicate that such efforts are especially effective. Another promising aspect is that these studies represent a range of developed and developing countries, thereby increasing the generalizability of the results. 


\subsection{Mining, Quarrying, and Oil and Gas Extraction}

Three studies were included, one each of RCT, CBA, and uCBA. Overall the results are encouraging as the RCT and uCBA demonstrated significant protective effects [32,60], while the CBA [34] showed promising effects in the protective direction (significance not stated). The relatively large effect sizes reported in these studies give us cautious optimism that education is an effective component in future mining interventions.

\subsection{Public Administration}

An RCT and an historically controlled study were included [28,44]. Both of which saw significant benefits in some but not all outcomes measured [Table 2]. Despite considerable cross-contamination between groups in the RCT, the authors persisted with an intention-to-treat (ITT) design which made the results more robust against non-adherence [28]. However, research in this sector is still lacking.

\subsection{Transportation and Warehousing}

One CBA and one uCBA were included [35,57]. Both studies only focused on a small subset of the overall population (i.e., stevedores and truck drivers with sleep disorders), which severely limits the generalizability of any conclusions drawn. As one of the most common causes of preventable fatal injuries, additional investigations in this field is needed [61].

\subsection{Utilities}

Salminen found an increase in injury numbers after implementing an anticipatory driving intervention for electricians [49]. In addition, although traffic-related incidents were reduced following another group discussion intervention, a paradoxical increase in non-traffic injury rates occurred. These could be rationalized by considering that the study was uncontrolled with a short follow-up period, and that behavioral changes may take a long time to mature. If the trial was more robust, the principles of driving safety could be extrapolated to other industries which involve driving. Another uCBA in the electrical sector was an abstract with relatively little information regarding injury outcomes, so it was not possible to draw conclusions from it [58]. Overall, educational interventions in this industry are understudied and greatly limited in scope.

\subsection{Observations across Industries}

Overall, the methodological quality of the included studies was poor, as shown in Figures 2 and 3. Even among the RCTs, none were at a low risk of bias. This observation can be rationalized by first considering the unique context of the occupational injury prevention scenario. Randomization in this setting may face ethical questions associated with denying potentially life-saving interventions from workers. In addition, even if that can be addressed, the study may run into logistical challenges when trying to recruit enough workplaces for randomization, as the prospect of being placed into the control group may discourage employers. On the other hand, if randomization was performed on an individual basis within individual workplaces, then serious concerns with cross-group contamination would be inevitable. Further, after randomization it would be practically and ethically unfeasible to prevent workplaces from implementing additional interventions, which would confound the results. This is especially pertinent with educational interventions, as changes in beliefs, attitudes, and work culture take time, during which confounding interventions may occur. Moreover, with an educational intervention, true blinding of the participants and instructors is practically challenging. For these reasons, sometimes the best available option is to implement a non-randomized trial or an RCT with considerable limitations. This has been echoed in previous Cochrane reviews [10,11]. As such, we have taken these constraints into account when making our recommendations.

To facilitate future RCTs, we suggest the following strategies. Conducting studies proactively (i.e., before injury rates become alarmingly high) could encourage employers to accept the risk of 
having their workplace being assigned to a control group. As an added benefit, it is less likely that employers will implement confounding interventions of their own during the study. Alternatively, offering crossover study designs would remove the disincentive of potentially being assigned to a control, while at the same time addressing ethical concerns of withholding beneficial interventions from workers. Finally, control groups may receive standard training or placebo intervention on another topic to enhance the blinding process. Such strategies could bring about additional high-quality studies in the field, which could in turn allow for further conclusions to be drawn.

There were four studies which attempted to indirectly prevent injuries through addressing associated conditions such as obesity, skin cancer, and sleep disorders [28,31,33,57]. Of which, Geller et al., Morgan et al., and Sullivan et al. were RCTs with significant results in at least some of their outcome measures. This suggests that education does not necessarily have to be directly focused on injuries. The fact that these three RCTs were performed in diverse settings supports the generalizability of this notion.

It should be noted that among the interventions which demonstrated a significant protective effect against occupational injuries, most featured either multifactorial strategies alongside education $[33,42,44,54,57]$ or educational approaches that were not purely didactic in nature $[30-32,38,52,55]$. This suggests that creative and multifaceted designs should be utilized when designing future injury prevention programs, especially in the manufacturing industry, where many of the aforementioned studies took place. While it may seem obvious that a multifactorial approach would have greater success than education alone, there is a potential synergistic effect as every link along the chain of safety is strengthened [64]. On the other hand, the effects of implementing didactic education on its own are limited, as evidenced by studies in the construction sector, which may not justify its resource and opportunity costs.

In general, developing countries were underrepresented amongst the included studies. Only six studies were done on workers in developing economies [30,32,35,54,55,58]. This is concerning, as the majority of occupational injuries occur in these settings [1]. Interestingly however, all of those studies showed either a significant benefit $[30,32,35,54,55]$ or a protective effect, without stating statistical significance [58], which suggests that education may be more effective in low-resource settings. This could be rationalized by considering that legislative, administrative, and engineering interventions presumably already exist in developed countries, thus the effects of additional education would be dwarfed in comparison. Conversely, in developing countries, those measures may not be as robust, which leaves the potential for education to impart a more pronounced effect.

\subsection{Limitations}

Due to limited resources, we were unable to include non-English language studies, which may have introduced a language bias. Additionally, since some educational interventions were implemented as part of a multifaceted program, it is sometimes difficult to discriminate what effects, if any, that the educational components truly imparted. However, this would not invalidate these studies as the purpose of our review is to address the pragmatic question of whether or not education is effective within the context of a real-life work environment, where there will inevitably be some degree of concurrent interventions. Due to heterogeneity in study designs and a limited number of RCTs, it was not possible for a meta-analysis to be performed and therefore quantitative conclusions cannot be drawn. Finally, since most studies were conducted in developed nations, a caveat is that our findings are not necessarily generalizable in the setting of developing economies.

\section{Conclusions}

Educational interventions are effective when implemented as part of a multifactorial approach or in a non-didactic fashion. This is especially true in the manufacturing sector. Caution should be advised when implementing didactic education on its own to prevent occupational injuries, especially in construction. Nevertheless, it could still be an effective component of a multifactorial approach. 
Additional high-quality studies in underrepresented industries and developing countries are needed to better understand the effectiveness of education in their respective settings. In the future, researchers could address barriers to RCTs by implementing interventions proactively, using crossover designs, and providing controls with standard training or placebo intervention.

Author Contributions: Conceptualization, J.L., J.S. and I.P.; methodology, J.L. and C.P.; validation, J.L., M.P. and I.P.; formal analysis, J.L.; investigation, J.L. and M.P.; resources, J.L. and I.P.; data curation, J.L.; writing-original draft preparation, J.L.; writing-review and editing, J.L., M.P., J.S., C.P. and I.P.; visualization, J.L.; supervision, J.S. and I.P.; project administration, J.L. and I.P.; funding acquisition, N/A. All authors have read and agreed to the published version of the manuscript.

Funding: This research received no external funding.

Acknowledgments: We would like to thank Alex Zheng who provided statistical advice to our systematic review. This research was supported by the facility accommodation of B.C. Injury Research and Prevention Unit.

Conflicts of Interest: The authors declare no conflict of interest.

\section{Appendix A}

We detailed the search strategy for Embase below. Modified forms of the search strategy were adopted for other electronic databases. All searches were conducted with the guidance of a biomedical librarian.

\#1. health education.mp. OR Health Education/OR primary prevention.mp. OR Primary Prevention/OR Health Promotion/OR social media.mp. OR Social Media/OR Marketing/OR Social Marketing/OR occupational health service.mp. OR Occupational Health Services/OR intervention.mp. OR educat*.mp. OR program*.mp.

\#2. work*.mp. OR occupation*.mp. OR employee.mp. OR industry.mp. OR Industry/OR agriculture.mp. OR Agriculture/OR farm*.mp. OR construction*.mp.

\#3. attitude*.mp. OR behavio*.mp. OR intention*.mp. OR belief*.mp. OR perception.mp. OR Perception/OR attitude to health.mp. OR Attitude to Health/OR Risk/OR Behavioral Risk Factor Surveillance System/OR Awareness/

\#4. occupational accident.mp. OR Accidents, Occupational/OR Insurance, Health/OR insurance claims.mp. OR absenteeism.mp. OR Absenteeism/OR Mortality/OR mortality risk.mp. OR (injur*adj3 reduction).mp. [ $\mathrm{mp}=$ title, abstract, heading word, drug trade name, original title, device manufacturer, drug manufacturer, device trade name, keyword, floating subheading word, candidate term word] OR (injur* adj3 prevention).mp. [mp = title, abstract, heading word, drug trade name, original title, device manufacturer, drug manufacturer, device trade name, keyword, floating subheading word, candidate term word] OR (accident* adj3 reduction).mp. [mp = title, abstract, heading word, drug trade name, original title, device manufacturer, drug manufacturer, device trade name, keyword, floating subheading word, candidate term word] OR (accident ${ }^{*}$ adj3 prevention).mp. [mp $=$ title, abstract, heading word, drug trade name, original title, device manufacturer, drug manufacturer, device trade name, keyword, floating subheading word, candidate term word]

\#5. wounds.mp. OR “Wounds and Injuries"/OR hazard*.mp. OR fatality.mp. OR disability.mp. OR accident.mp. OR Accidents/OR death*.mp. OR injur*mp.

\#6. \#1 AND \#2 AND \#3 AND \#4 AND \#5 


\section{Appendix B}

Table A1. The reasons for exclusion of all studies that were excluded after a full-text review are listed below.

\begin{tabular}{|c|c|}
\hline Study & Reason(s) for Exclusion \\
\hline $\begin{array}{l}\text { Bahn S, Barratt-Pugh L. Evaluation of the mandatory } \\
\text { construction induction training program in Western } \\
\text { Australia: unanticipated consequences. Eval Program } \\
\text { Plann. 2012; 35(3): 337-43. [65] }\end{array}$ & Injury outcomes were not quantified \\
\hline $\begin{array}{l}\text { Sarma BP. Prevention of burns: } 13 \text { years' experience in } \\
\text { Northeastern India. Burns. 2011;37(2): 265-72. [66] }\end{array}$ & $\begin{array}{c}\text { Interventions and outcomes are not specific to the } \\
\text { occupational setting }\end{array}$ \\
\hline $\begin{array}{l}\text { de Boer AGEM, Burdorf A, van Duivenbooden C, } \\
\text { Frings-Dresen MHW. The effect of individual counselling } \\
\text { and education on work ability and disability pension: a } \\
\text { prospective intervention study in the construction } \\
\text { industry. Occup Environ Med. 2007; 64(12): 792-7. [67] }\end{array}$ & $\begin{array}{l}\text { The study measured disability as an outcome, but it } \\
\text { didn't specify if this referred to disability due to acute } \\
\text { injuries, chronic exertion, or non-physical causes }\end{array}$ \\
\hline $\begin{array}{l}\text { Donham KJ, Lange JL, Kline A, Rautiainen RH, Grafft L. } \\
\text { Prevention of occupational respiratory symptoms among } \\
\text { certified safe farm intervention participants. J } \\
\text { Agromedicine. 2010; 16(1): 40-51. [68] }\end{array}$ & $\begin{array}{l}\text { The study only measured organic dust toxic } \\
\text { syndrome, which should be classified as an } \\
\text { occupational disease instead of occupational injury }\end{array}$ \\
\hline $\begin{array}{l}\text { Donham KJ, Rautiainen RH, Lange JL, Schneiders S. } \\
\text { Injury and illness costs in the Certified Safe Farm study. J } \\
\text { Rural Health. 2007; 23(4): 348-55. [69] }\end{array}$ & Same study as Rautiainen et al. 2004 [12] \\
\hline $\begin{array}{l}\text { Hagel LM, Pickett W, Pahwa P, Day L, Brison RJ, } \\
\text { Marlenga B, et al. Prevention of agricultural injuries: an } \\
\text { evaluation of an education-based intervention. Inj Prev. } \\
\text { 2008 Oct } 1 ; 14(5): 290-5 .[70]\end{array}$ & $\begin{array}{l}\text { This was a cross-sectional survey, which was not one } \\
\text { of the study types listed in our inclusion criteria }\end{array}$ \\
\hline $\begin{array}{l}\text { Melamed S, Oksenberg A. Excessive daytime sleepiness } \\
\text { and risk of occupational injuries in non-shift daytime } \\
\text { workers. Sleep. 2002; 25(3): 315-21. [71] }\end{array}$ & Did not provide sufficient injury outcome data \\
\hline $\begin{array}{l}\text { Taylor EL. Safety benefits of mandatory OSHA } 10 \mathrm{~h} \\
\text { training. Saf Sci. 2015; 77: 66-71. [72] }\end{array}$ & Legislative intervention \\
\hline $\begin{array}{c}\text { Trinh MT. Developing Resilient Safety Culture for } \\
\text { Construction Projects in Vietnam. Western Sydney } \\
\text { University (Australia); 2018. [73] }\end{array}$ & Not an educational intervention \\
\hline $\begin{array}{l}\text { Vieira F. Importance of the sleepiness and fatigue } \\
\text { prevention for reducing occupational accidents in a } \\
\text { Brazilian mining company. Sleep Med. 2011; 12(Suppl 1): } \\
\text { S21-2. [74] }\end{array}$ & $\begin{array}{l}\text { The intervention has very few educational } \\
\text { components, which are also vaguely defined. } \\
\text { Moreover, the study only measured equipment } \\
\text { downtime and unmet production due to drowsiness, } \\
\text { which are not sufficiently reliable outcomes according } \\
\text { to our inclusion criteria }\end{array}$ \\
\hline $\begin{array}{l}\text { Young S. From zero to hero. A case study of industrial } \\
\text { injury reduction: New Zealand Aluminium Smelters } \\
\text { Limited. Saf Sci. 2014; 64: 99-108. [75] }\end{array}$ & $\begin{array}{c}\text { The behavioral component is a very small proportion } \\
\text { of the intervention and it's not consistently } \\
\text { implemented }\end{array}$ \\
\hline
\end{tabular}

Yu W. Evaluating the Effectiveness of Participatory

Training for Occupational Health and Safety

Improvements-A Randomized Controlle Trial with

One-year Follow-up in China. ProQuest Dissertations

Same study as Yu et al. 2017 [30]

and Theses. The Chinese University of Hong Kong

(Hong Kong); 2010. [76]

Yu I, Yu W, Li Z. The effectiveness of participatory

training on reduction of occupational injuries:

A randomised controlled trial. Occup Environ Med. 2011;

Same study as Yu et al. 2017 [30] 68(Suppl 1): S24-5. [77] 


\section{Appendix C}

Table A2. Author disclosures for funding and conflicts of interests (COI) were searched for all included studies.

\begin{tabular}{|c|c|}
\hline Study ID & Disclosures \\
\hline Rasmussen et al., 2003 [26] & $\begin{array}{l}\text { Funded by grants from the Danish Insurance Association, The Work } \\
\text { Environment Fund, The Health Fund of the Danish Ministry of Health, } \\
\text { and the Public Health Research Fund of the counties of Ribe and } \\
\text { Ringkoebing, Denmark. COI information not provided }\end{array}$ \\
\hline Vercruysse et al., 2016 [27] & $\begin{array}{c}\text { Supported by Flemish Policy Research Centre on Sports; authors declared } \\
\text { no COI }\end{array}$ \\
\hline Sullivan et al., 2017 [28] & $\begin{array}{l}\text { Funded by Federal Emergency Management Agency (FEMA) Assistance } \\
\text { for Firefighters grants EMW-2007-FP-02197 and EMW-2008-FP-02566; } \\
\text { National Institute of Occupational Safety and Health grant } \\
\text { 1R01OH010300-02; National Institutes of Health National Heart, Lung, } \\
\text { and Blood Institute grant R01NS054277; and an endowed professorship } \\
\text { provided to Harvard Medical School by Cephalon, Inc. Some researchers } \\
\text { had lecture fees, previous research support, association with various } \\
\text { organizations, and consulting histories }\end{array}$ \\
\hline Rautiainen et al., 2004 [29] & $\begin{array}{l}\text { The Certified Safe Farm study was funded by NIOSH (Grant No. } \\
\text { U06/CCU712913), the Iowa Wellmark Foundation, Pioneer Hi-Bred } \\
\text { International, Inc., the Iowa Pork Producers Council, the National Pork } \\
\text { Producer's Council, and Iowa's Injury Prevention Research Center. COI } \\
\text { information not provided }\end{array}$ \\
\hline Yu et al., 2017 [30] & $\begin{array}{c}\text { Funded by the Chinese University of Hong Kong, the Hong Kong } \\
\text { Workers' Health Center and the Shenzhen Hospital for Occupational } \\
\text { Disease Control and Prevention; authors declared no COI }\end{array}$ \\
\hline Morgan et al., 2012 [31] & $\begin{array}{l}\text { Supported by the Hunter Medical Research Institute and funded by } \\
\text { Tomago Aluminium and the Hunter Medical Research Institute. Tomago } \\
\text { had no involvement in study design, analysis, and interpretation of data, } \\
\text { or the decision to submit the manuscript for publication. S.M. from } \\
\text { Tomago Aluminium reviewed the drafted manuscript for accuracy and } \\
\text { also organized the data collection at Tomago and assisted with recruitment }\end{array}$ \\
\hline Adams et al., 2013 [32] & $\begin{array}{l}\text { This trial was funded by an intra-mural research grant from the Fluid } \\
\text { Research Fund of the Christian Medical College, Vellore, administered } \\
\text { through the Office of Research. Protective eyewear was funded by a } \\
\text { project grant from the Christoffel-Blindenmission (CBM) to the } \\
\text { Department of Ophthalmology, Christian Medical College, Vellore. } \\
\text { The funding sources had no role in the design, conduct, or reporting of } \\
\text { this trial; authors declared no COI }\end{array}$ \\
\hline Geller et al., 2001 [33] & $\begin{array}{l}\text { Centers for Disease Control and Prevention, Grant U56-CCU 914658. No } \\
\text { info on COI }\end{array}$ \\
\hline Hagge et al., 2016 [34] & None provided \\
\hline Robaina et al., 2010 [35] & $\begin{array}{l}\text { "Drs. Robaina and Ávila are members of the Scientific Council of the } \\
\text { National Institute of Workers' Health, Cuba. Dr. Partanen declares no } \\
\text { conflicts of interest." }\end{array}$ \\
\hline Hermann et al., 2010 [36] & None provided \\
\hline Mancini et al., 2005 [37] & No information on funding, no competing interests \\
\hline Yeow et al., 2014 [38] & None provided \\
\hline Kidd et al., 2000 [39] & Grant-Number-R01-CCR-413067 \\
\hline Borger et al., 2011 [40] & None provided \\
\hline Bena et al., 2009 [41] & $\begin{array}{c}\text { This work was supported by a grant from the Regione Piemonte. COI not } \\
\text { provided }\end{array}$ \\
\hline
\end{tabular}


Table A2. Cont.

\begin{tabular}{|c|c|}
\hline Study ID & Disclosures \\
\hline Porru et al., 2011 [42] & Authors declared no COI, no information about grants \\
\hline Porru et al., 2017 [43] & $\begin{array}{c}\text { Association of Italian Foundries (ASSOFOND) provided "assistance and } \\
\text { support." Otherwise no COI or grants mentioned. }\end{array}$ \\
\hline Knapik et al., 2004 [44] & None provided \\
\hline Boini et al., 2017 [45] & $\begin{array}{l}\text { This research received no specific grant from any funding agency in the } \\
\text { public, commercial or non-profit sectors; authors declared no COI }\end{array}$ \\
\hline Schoenfisch et al., 2017 [46] & $\begin{array}{c}\text { Funding by CPWR-The Center for Construction Research and } \\
\text { Training-through cooperative agreement number U60-OH009762 from the } \\
\text { National Institute of Occupational Safety and Health (NIOSH). Authors } \\
\text { declared no COI. }\end{array}$ \\
\hline Koblesky et al., 2015 [47] & Author declared no grants COI and no commercial COI \\
\hline Gatti et al., 2013 [48] & None provided \\
\hline Williams et al., 2010 [53] & $\begin{array}{c}\text { Funded by the Center for Construction Research and Training - CPWR } \\
\text { through a grant from NIOSH. The lead author was supported in part by } \\
\text { the Center for Disease Control and Prevention (CDC), Training Program } \\
\text { Grant \# } 5 \text { T01 CD000189-05. }\end{array}$ \\
\hline Shouman et al., 2002 [54] & None provided \\
\hline Nunu et al., 2018 [55] & $\begin{array}{c}\text { Authors declared no "significant financial support... that could have } \\
\text { influenced its outcome" }\end{array}$ \\
\hline Day 2002 [56] & $\begin{array}{l}\text { None provided, except that the lead author worked at the mill of } \\
\text { intervention }\end{array}$ \\
\hline Howard et al., 2009 [57] & $\begin{array}{c}\text { Funding: Worksafe Victoria, Transport Accident Commission. No } \\
\text { information on conflicts of interest }\end{array}$ \\
\hline Badmos et al., 2018 [58] & None provided \\
\hline Johnson et al., 2002 [59] & None provided \\
\hline $\begin{array}{l}\text { Kowalski- Trakofler et al., } \\
2003 \text { [60] }\end{array}$ & None provided \\
\hline Salminen 2008 [49] & None provided \\
\hline Evanoff et al., 2016 [50] & $\begin{array}{l}\text { Funding by the Center for Construction Research and Training through } \\
\text { the National Institute of Occupational Safety and Health (U54 OH00830 } \\
\text { and U60 OH009762) and the Washington University Institute of Clinical } \\
\text { and Translational Sciences grant UL1 TR000448 from the National Center } \\
\text { for Advancing Translational Sciences (NCATS) of the National Institutes } \\
\text { of Health (NIH). Authors declared no financial COI }\end{array}$ \\
\hline Darragh et al., 2004 [51] & $\begin{array}{c}\text { Funding by the US Department of Labor, Occupational Safety and Health } \\
\text { Administration (OSHA) through a cooperative agreement with the } \\
\text { Technical Assistance and Training Corporation, Washington, DC. No info } \\
\text { on COI }\end{array}$ \\
\hline Spangenberg et al., 2002 [52] & $\begin{array}{c}\text { Commissioned by the owner of the construction site (A/S } \\
\text { Øresundsforbindelsen) }\end{array}$ \\
\hline
\end{tabular}

\section{References}

1. Hämäläinen, P.; Takala, J.; Kiat, T.B. Global Estimates of Occupational Accidents and Work-Related Illnesses 2017; Workplace Safety and Health Institute: Singapore, 2017.

2. Takala, J.; Hämäläinen, P.; Saarela, K.L.; Yun, L.Y.; Manickam, K.; Jin, T.W.; Heng, P.; Tjong, C.; Kheng, L.G.; Lim, S. Global estimates of the burden of injury and illness at work in 2012. J. Occup. Environ. Hyg. 2014, 11, 326-337. [CrossRef] [PubMed] 
3. International Labour Organization Global Trends on Occupational Accidents and Diseases. Available online: https://www.ilo.org/legacy/english/osh/en/story_content/external_files/fs_st_1-ILO_5_en.pdf (accessed on 14 August 2019).

4. Pike, I.; Richmond, S.; Rothman, L.; Macpherson, A. (Eds.) Canadian Injury Prevention Resource; Parachute: Toronto, ON, Canada, 2015.

5. Lund, J.; Aarø, L.E. Accident prevention. Presentation of a model placing emphasis on human, structural and cultural factors. Saf. Sci. 2004, 42, 271-324. [CrossRef]

6. Munro, J.; Coleman, P.; Nicholl, J.; Harper, R.; Kent, G.; Wild, D. Can we prevent accidental injury to adolescents? A systematic review of the evidence. Inj. Prev. 1995, 1, 249-255. [CrossRef] [PubMed]

7. DeRoo, L.A.; Rautiainen, R.H. A systematic review of farm safety interventions. Am. J. Prev. Med. 2000, 18, 51-62. [CrossRef]

8. McCallum, D.M.; Murphy, S.; Reed, D.B.; Claunch, D.T.; Reynolds, S.J. What we know about the effectiveness of farm safety day programs and what we need to know. J. Rural Heal. 2013, 29, 20-29. [CrossRef]

9. Ruotsalainen, J.H.; Verbeek, J.H.; Salmi, J.A.; Jauhiainen, M.; Laamanen, I.; Pasternack, I.; Husman, K. Evidence on the effectiveness of occupational health interventions. Am. J. Ind. Med. 2006, 49, 865-872. [CrossRef]

10. Van der Molen, H.F.; Lehtola, M.M.; Lappalainen, J.; Hoonakker, P.L.T.; Hsiao, H.; Haslam, R.; Hale, A.R.; Frings-Dresen, M.H.W.; Verbeek, J.H. Interventions to prevent injuries in construction workers. Cochrane database Syst. Rev. 2012, 12, CD006251. [CrossRef]

11. Rautiainen, R.; Lehtola, M.M.; Day, L.M.; Schonstein, E.; Suutarinen, J.; Salminen, S.; Verbeek, J.H. Interventions for preventing injuries in the agricultural industry. Cochrane Database Syst. Rev. 2008. [CrossRef]

12. Moher, D.; Liberati, A.; Tetzlaff, J.; Altman, D.G.; Group, P. Preferred reporting items for systematic reviews and meta-analyses: The PRISMA statement. PLoS Med. 2009, 6, e1000097. [CrossRef]

13. Non-randomised Controlled Study (NRS) Designs. Available online: https://childhoodcancer.cochrane.org/ non-randomised-controlled-study-nrs-designs (accessed on 8 May 2019).

14. Baker, S.P.; Ginsburg, M.J.; O’Neill, B.; Li, G. The Injury Fact Book; Oxford University Press: Oxford, UK, 1992; ISBN 0195061942.

15. Peden, M.; McGee, K.; Sharma, G. The Injury Chart Book: A Graphical Overview of the Global Burden of Injuries; World Health Organization: Geneva, Switzerland, 2002; ISBN 924156220X.

16. Katz, L.F.; Margo, R.A. Technical change and the relative demand for skilled labor: The united states in historical perspective. In Human Capital in History: The American Record; University of Chicago Press: Chicago, IL, USA, 2014; pp. 15-57.

17. David, H. Why are there still so many jobs? The history and future of workplace automation. J. Econ. Perspect. 2015, 29, 3-30.

18. Committee to Assess Training Needs for Occupational Safety and Health Personnel in the United States. Safe Work in the 21st Century: Education and Training Needs for the Next decade's Occupational Safety and Health Personnel; National Academies Press: Washington, DC, USA, 2000; ISBN 0309070260.

19. Cox, A.R.; Williamson, G.C. Job security for occupational health and safety professionals in the 21st century: What you need to know about the Institute of Medicine (2000) report. Aaohn J. 2001, 49, 169-170. [CrossRef] [PubMed]

20. Scherer, R.W.; Langenberg, P.; Von Elm, E. Full publication of results initially presented in abstracts. Cochrane Database Syst. Rev. 2005. [CrossRef]

21. North American Industry Classification System (NAICS) Canada 2017 Version 1.0. Available online: http://www23.statcan.gc.ca/imdb/p3VD.pl?Function=getVD\&TVD=307532 (accessed on 9 May 2019).

22. Sterne, J.A.C.; Savović, J.; Page, M.J.; Elbers, R.G.; Blencowe, N.S.; Boutron, I.; Cates, C.J.; Cheng, H.-Y.; Corbett, M.S.; Eldridge, S.M. RoB 2: A revised tool for assessing risk of bias in randomised trials. BMJ 2019, 366, 14898. [CrossRef] [PubMed]

23. Sterne, J.A.C.; Hernán, M.A.; Reeves, B.C.; Savović, J.; Berkman, N.D.; Viswanathan, M.; Henry, D.; Altman, D.G.; Ansari, M.T.; Boutron, I. ROBINS-I: A tool for assessing risk of bias in non-randomised studies of interventions. BMJ 2016, 355, I4919. [CrossRef]

24. Higgins, J.; Green, S. (Eds.) Cochrane Handbook for Systematic Reviews of Interventions; The Cochrane Collaboration: London, UK, 2011. 
25. National Safety Council Industry Incidence and Rates-Industry Profiles. Available online: https://injuryfacts. nsc.org/work/industry-incidence-rates/industry-profiles/ (accessed on 28 July 2019).

26. Rasmussen, K.; Carstensen, O.; Lauritsen, J.M.; Glasscock, D.J.; Hansen, O.N.; Jensen, U.F. Prevention of farm injuries in Denmark. Scand. J. Work. Environ. Heal. 2003, 29, 288-296. [CrossRef] [PubMed]

27. Vercruysse, S.; Haerens, L.; Verhagen, E.; Goossens, L.; De Clercq, D. Effects of a multifactorial injury prevention intervention in physical education teachers: A randomized controlled trial. Eur. J. Sport Sci. 2016, 16, 868-876. [CrossRef] [PubMed]

28. Sullivan, J.P.; O’Brien, C.S.; Barger, L.K.; Rajaratnam, S.M.W.; Czeisler, C.A.; Lockley, S.W.; Harvard Work Hours, Health and Safety Group. Randomized, prospective study of the impact of a sleep health program on firefighter injury and disability. Sleep 2017, 40, zsw001. [CrossRef]

29. Rautiainen, R.H.; Lange, J.L.; Hodne, C.J.; Schneiders, S.; Donham, K.J. Injuries in the Iowa certified safe farm study. J. Agric. Saf. Health 2004, 10, 51-63. [CrossRef]

30. Yu, I.T.S.; Yu, W.; Li, Z.; Qiu, H.; Wan, S.; Xie, S.; Wang, X. Effectiveness of participatory training in preventing accidental occupational injuries: A randomized-controlled trial in China. Scand. J. Work. Environ. Health 2017, 43, 226-233. [CrossRef]

31. Morgan, P.J.; Collins, C.E.; Plotnikoff, R.C.; Cook, A.T.; Berthon, B.; Mitchell, S.; Callister, R. The impact of a workplace-based weight loss program on work-related outcomes in overweight male shift workers. J. Occup. Environ. Med. 2012, 54, 122-127. [CrossRef]

32. Adams, J.S.K.; Raju, R.; Solomon, V.; Samuel, P.; Dutta, A.K.; Rose, J.S.; Tharyan, P. Increasing compliance with protective eyewear to reduce ocular injuries in stone-quarry workers in Tamil Nadu, India: A pragmatic, cluster randomised trial of a single education session versus an enhanced education package delivered over six months. Injury 2013, 44, 118-125. [CrossRef] [PubMed]

33. Geller, A.C.; Glanz, K.; Shigaki, D.; Isnec, M.R.; Sun, T.; Maddock, J. Impact of skin cancer prevention on outdoor aquatics staff: The Pool Cool program in Hawaii and Massachusetts. Prev Med 2001, 33, $155-161$. [CrossRef]

34. Hagge, M.; McGee, H.; Matthews, G.; Aberle, S. Behavior-based safety in a coal mine: The relationship between observations, participation, and injuries over a 14-year period. J. Organ. Behav. Manage. 2017, 37, 107-118. [CrossRef]

35. Robaina, C.; Partanen, T.J.; Avila, I. A program for the reduction of occupational injuries and changes in safety culture among stevedores at Port of Havana, Cuba. Int. J. Occup. Environ. Health 2010, 16, 312-319. [CrossRef]

36. Hermann, J.A.; Ibarra, G.V.; Hopkins, B.L. A safety program that integrated behavior-based safety and traditional safety methods and its effects on injury rates of manufacturing workers. J. Organ. Behav. Manage. 2010, 30, 6-25. [CrossRef]

37. Mancini, G.; Baldasseroni, A.; Laffi, G.; Curti, S.; Mattioli, S.; Violante, F.S. Prevention of work related eye injuries: Long term assessment of the effectiveness of a multicomponent intervention among metal workers. Occup. Environ. Med. 2005, 62, 830-835. [CrossRef]

38. Yeow, P.H.P.; Goomas, D.T. Outcome-and-behavior-based safety incentive program to reduce accidents: A case study of a fluid manufacturing plant. Saf. Sci. 2014, 70, 429-437. [CrossRef]

39. Kidd, P.; Struttmann, T.; Parshall, M.; Wojcik, S. Loss Education to Reduce Construction-Related Injuries; Kentucky Injury Prevention and Research Center: Atlanta, GA, USA, 2000.

40. Borger, J.; Sun, Y.; Bochmann, F.; Guldner, K.; Ponto, K. Reduction of occupational injuries by conduction of a preventive training programme - An epidemiological follow-up study in the German glass industry. Occup. Environ. Med. 2011, 68, S25. [CrossRef]

41. Bena, A.; Berchialla, P.; Coffano, M.E.; Debernardi, M.L.; Icardi, L.G. Effectiveness of the training program for workers at construction sites of the high-speed railway line between Torino and Novara: Impact on injury rates. Am. J. Ind. Med. 2009, 52, 965-972. [CrossRef]

42. Porru, S.; Calza, S.; Arici, C. An effectiveness evaluation of a multifaceted preventive intervention on occupational injuries in foundries: A 13-year follow-up study with interrupted time series analysis. Int. Arch. Occup. Environ. Health 2011, 84, 867-876. [CrossRef]

43. Porru, S.; Calza, S.; Arici, C. Prevention of occupational injuries: Evidence for effective good practices in foundries. J. Safety Res. 2017, 60, 53-69. [CrossRef] [PubMed] 
44. Knapik, J.J.; Bullock, S.H.; Toney, E.; Wells, J.D.; Hoedebecke, E.; Jones, B.H. Influence of an injury reduction program on injury and fitness outcomes among soldiers. Inj. Prev. 2004, 10, 37-42. [CrossRef]

45. Boini, S.; Colin, R.; Grzebyk, M. Effect of occupational safety and health education received during schooling on the incidence of workplace injuries in the first 2 years of occupational life: A prospective study. BMJ Open 2017, 7, e015100. [CrossRef]

46. Schoenfisch, A.L.; Lipscomb, H.; Sinyai, C.; Adams, D. Effectiveness of OSHA Outreach Training on carpenters' work-related injury rates, Washington State 2000-2008. Am. J. Ind. Med. 2017, 60, 45-57. [CrossRef] [PubMed]

47. Koblesky, N. Workers compensation E-Mod factor improves through actionable measures. Transfusion 2015, 55, S228-S229.

48. Gatti, M.; Valdina, A.; Tosolin, F.; Arezes, P.; Baptista, J.S.; Barroso, M.P.; Carneiro, P.; Costa, N.; Melo, R.; Miguel, A.S.; et al. The reduction of injuries due to the Behavior-Based Safety processes implemented in several plants and sites A collection of case studies reviews the results obtained from behavior analysis applied to industrial environment. In Proceedings of the Occupational Safety and Hygiene, Guimarães, Portugal, 12 February 2013; pp. 216-218.

49. Salminen, S. Two interventions for the prevention of work-related road accidents. Saf. Sci. 2008, 46, 545-550. [CrossRef]

50. Evanoff, B.; Dale, A.; Zeringue, A.; Fuchs, F.; Gaal, J.; Lipscomb, H.J. Results of a fall prevention educational intervention for residential construction. Saf. Sci. 2016, 89, 301-307. [CrossRef]

51. Darragh, A.R.; Stallones, L.; Bigelow, P.L.; Keefe, T.J. Effectiveness of the HomeSafe Pilot Program in reducing injury rates among residential construction workers, 1994-1998. Am. J. Ind. Med. 2004, 45, 210-217. [CrossRef]

52. Spangenberg, S.; Mikkelsen, K.L.; Kines, P.; Dyreborg, J. The construction of the Oresund link between Denmark and Sweden: The effect of a multi-faceted safety campaign. Saf. Sci. 2002, 40, 457-465. [CrossRef]

53. Williams, Q., Jr.; Ochsner, M.; Marshall, E.; Kimmel, L.; Martino, C. The impact of a peer-led participatory health and safety training program for Latino day laborers in construction. J. Safety Res. 2010, 41, $253-261$. [CrossRef]

54. Shouman, A.E.; El-Damaty, S.I.; Bakr, I.; Mukhtar, A.; Kamal, A.M. Accident prevention program in a glass factory in Shoubra El Khema district. J. Egypt. Public Health Assoc. 2002, 77, 73-99. [PubMed]

55. Nunu, W.; Tendai, k.; Phakamani, M. An evaluation of the effectiveness of the Behaviour Based Safety Initiative card system at a cement manufacturing company in Zimbabwe. Saf. Health Work 2018, 9, 308-313. [CrossRef] [PubMed]

56. Day, R.C. Changing a Corporate Culture: Implementing Safety Leadership at a Pulp and Paper mill; St. Francis Xavier University: Ann Arbor, MI, USA, 2002.

57. Howard, M.; Wilson, J.; Hare, D.; Swann, P. Health break - Sleep disorders screening in road transport. Sleep Biol. Rhythms 2009, 7, S14. [CrossRef]

58. Badmos, E. Accident prevention: From vision to action through "harm to zero" concept. Occup. Environ. Med. 2018, 75, S31. [CrossRef]

59. Johnson, K.A.; Ruppe, J. A job safety program for construction workers designed to reduce the potential for occupational injury using tool box training sessions and computer-assisted biofeedback stress management techniques. Int. J. Occup. Saf. Ergon. 2002, 8, 321-329. [CrossRef] [PubMed]

60. Kowalski-Trakofler, K.M.; Barrett, E.A. The concept of degraded images applied to hazard recognition training in mining for reduction of lost-time injuries. J. Safety Res. 2003, 34, 515-525. [CrossRef] [PubMed]

61. National Safety Council Work Safety Introduction. Available online: https://injuryfacts.nsc.org/work/workoverview/work-safety-introduction/ (accessed on 11 July 2019).

62. The World Bank World Development Report 2008: Agriculture for Development. Available online: http: //siteresources.worldbank.org/INTWDRS/Resources/477365-1327599046334/WDR_00_book.pdf (accessed on 11 July 2019).

63. Lipscomb, H.J.; Li, L.; Dement, J. Work-related falls among union carpenters in Washington State before and after the Vertical Fall Arrest Standard. Am. J. Ind. Med. 2003, 44, 157-165. [CrossRef]

64. Cagno, E.; Micheli, G.J.L.; Jacinto, C.; Masi, D. An interpretive model of occupational safety performance for Small-and Medium-sized Enterprises. Int. J. Ind. Ergon. 2014, 44, 60-74. [CrossRef]

65. Bahn, S.; Barratt-Pugh, L. Evaluation of the mandatory construction induction training program in Western Australia: Unanticipated consequences. Eval. Program Plann. 2012, 35, 337-343. [CrossRef] 
66. Sarma, B.P. Prevention of burns: 13 years' experience in Northeastern India. Burns 2011, 37, $265-272$. [CrossRef]

67. De Boer, A.G.E.M.; Burdorf, A.; van Duivenbooden, C.; Frings-Dresen, M.H.W. The effect of individual counselling and education on work ability and disability pension: A prospective intervention study in the construction industry. Occup. Environ. Med. 2007, 64, 792-797. [CrossRef] [PubMed]

68. Donham, K.J.; Lange, J.L.; Kline, A.; Rautiainen, R.H.; Grafft, L. Prevention of occupational respiratory symptoms among certified safe farm intervention participants. J. Agromedicine 2010, 16, 40-51. [CrossRef] [PubMed]

69. Donham, K.J.; Rautiainen, R.H.; Lange, J.L.; Schneiders, S. Injury and illness costs in the Certified Safe Farm study. J. Rural Health 2007, 23, 348-355. [CrossRef] [PubMed]

70. Hagel, L.M.; Pickett, W.; Pahwa, P.; Day, L.; Brison, R.J.; Marlenga, B.; Crowe, T.; Snodgrass, P.; Ulmer, K.; Dosman, J.A. Prevention of agricultural injuries: An evaluation of an education-based intervention. Inj. Prev. 2008, 14, 290-295. [CrossRef] [PubMed]

71. Melamed, S. Excessive daytime sleepiness and risk of occupational injuries in non-shift daytime workers. Sleep 2002, 25, 315-321. [CrossRef]

72. Taylor, E.L. Safety benefits of mandatory OSHA $10 \mathrm{~h}$ training. Saf. Sci. 2015, 77, 66-71. [CrossRef]

73. Trinh, M.T. Developing Resilient Safety Culture for Construction Projects in Vietnam; Western Sydney University (Australia): Sydney, NSW, Australia, 2018.

74. Vieira, F. Importance of the sleepiness and fatigue prevention for reducing occupational accidents in a Brazilianmining company. Sleep Med. 2011, 12, S21-S22. [CrossRef]

75. Young, S. From zero to hero. A case study of industrial injury reduction: New Zealand Aluminium Smelters Limited. Saf. Sci. 2014, 64, 99-108. [CrossRef]

76. Yu, W. Evaluating the Effectiveness of Participatory Training for Occupational Health and Safety Improvements-A Randomized Controlle Trial with One-year Follow-up in China; The Chinese University of Hong Kong (Hong Kong): Hong Kong, China, 2010.

77. Yu, I.; Yu, W.; Li, Z. The effectiveness of participatory training on reduction of occupational injuries: A randomised controlled trial. Occup. Environ. Med. 2011, 68, S24-S25. [CrossRef] 